\title{
Enhancing the Linearity Characteristics of Photoelectric Displacement Sensor Based on Extreme Learning Machine Method
}

\author{
Murugan SETHURAMALINGAM ${ }^{1}$ and Umayal SUBBIAH ${ }^{2}$ \\ ${ }^{1}$ Department of Electrical and Electronics Engineering, Einstein College of Engineering affiliated to Anna University, \\ Chennai, Tamil Nadu, India \\ ${ }^{2}$ Department of Electrical and Electronics Engineering, Ultra College of Engineering and Technology for Women \\ affiliated to Anna University, Chennai, Tamil Nadu, India \\ ${ }^{*}$ Corresponding author: Murugan SETHURAMALINGAM E-mail: srivimurugan@gmail.com
}

\begin{abstract}
Photoelectric displacement sensors rarely possess a perfectly linear transfer characteristic, but always have some degree of non-linearity over their range of operation. If the sensor output is nonlinear, it will produce a whole assortment of problems. This paper presents a method to compensate the nonlinearity of the photoelectric displacement sensor based on the extreme learning machine (ELM) method which significantly reduces the amount of time needed to train a neural network with the output voltage of the optical displacement sensor and the measured input displacement to eliminate the nonlinear errors in the training process. The use of this proposed method was demonstrated through computer simulation with the experimental data of the sensor. The results revealed that the proposed method compensated the presence of nonlinearity in the sensor with very low training time, lowest mean squared error (MSE) value, and better linearity. This research work involved less computational complexity, and it behaved a good performance for nonlinearity compensation for the photoelectric displacement sensor and has a good application prospect.
\end{abstract}

Keywords: Photoelectric displacement sensor, nonlinearity, extreme learning machine method

Citation: Murugan SETHURAMALINGAM and Umayal SUBBIAH, "Enhancing the Linearity Characteristics of Photoelectric Displacement Sensor Based on Extreme Learning Machine Method," Photonic Sensors, 2015, 5(1): 24-31.

\section{Introduction}

The photoelectric displacement sensor is widely used in the on-line measurement for the boundary position in the industrial production and experiments such as steel rolling, textile, and printing. It is also used to ensure the successful completion of the tape, trimming border, and overprinting pattern or to ensure a better damping effect and vibration isolation. In theory, the relationship between the input displacement and output voltage of the optical displacement sensor [1-3] is non-linear. The input-output data from the optical measurement system must be corrected to improve the measurement accuracy. In the practical application, piecewise linear correction methods are commonly used. However, they are usually short of preciseness in some situations that require higher precision. In order to improve the measurement accuracy of photoelectric sensors, researchers have made great efforts by various experimental and digital correction methods [4-11]. But the phenomenon of inadequate precision still exists, which cannot meet the actual needs for testing high precision displacement parameters. The support vector machine (SVM) is a new machine learning method

Received: 14 September 2014 / Revised version: 7 December 2014

(C) The Author(s) 2014. This article is published with open access at Springerlink.com DOI: $10.1007 / \mathrm{s} 13320-014-0219-7$

Article type: Regular 
[12-15], which is established on the statistical learning theory, based on the structural risk minimization principle, and possesses a good generalization ability. As SVM algorithm is a convex quadratic optimization problem, it can guarantee to find the global optimal solution and can solve the problem with the small sampling number, nonlinear relation, and high dimension. To overcome the difficulties faced by the above said techniques, the extreme learning machine (ELM), a nonlinear compensation method was proposed in this work, which could make the optical displacement sensor linear. The corrected network could be processed according to the linear characteristics, and the measurement accuracy was improved.

This paper is organized as follows: after introduction in Section 1, a brief description on the photoelectric displacement sensor is given in Section 2. Experimental observations of the sensor are also discussed in this section. Section 3 deals with the mathematical analysis of the ELM. The computer simulation study of the proposed models by using the experimental data of the optical sensor are carried out in this section. Results and discussion with output performance curves before and after compensation of nonlinearity using the specified algorithms are mentioned in Section 4. Finally conclusions and future scopes are discussed in Section 5 .

\section{Photoelectric displacement sensor}

\subsection{Principle of operation}

The photoelectric displacement sensor is a lateral semiconductor-based photo-detector device. The structure and equivalent circuit of the sensor are shown in Figs. 1 and 2, respectively. It is a non-split-type photoelectric conversion device in a position to continuously detect the light point. The practical optical displacement sensor generally uses the positive-intrinsic-negative (PIN) semiconductor structure, as shown in Fig. 1. Its surface is P layer on the light-sensitive side with the output electrode on each side, the middle is I layer, and the bottom layer is highly doped $\mathrm{N}$ layer, to reverse the bias voltage. When the incident light reaches a point of the photosensitive surface of the optical displacement sensor, photo-generated carriers are excited after the photon absorption by the semiconductor. Under transverse electric fields, carriers flow to both ends of the output electrode, forming the output current $I_{1}$ and $I_{2}$, and the ratio of $I_{1}$ to $I_{2}$ will change with the movement of the light source position. The location of points of light could be determined according to the current signal proportion collected on electrodes.

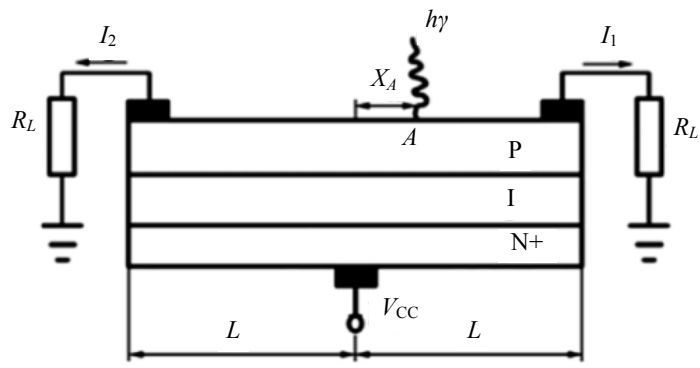

Fig. 1 Structure of the photoelectric displacement sensor.

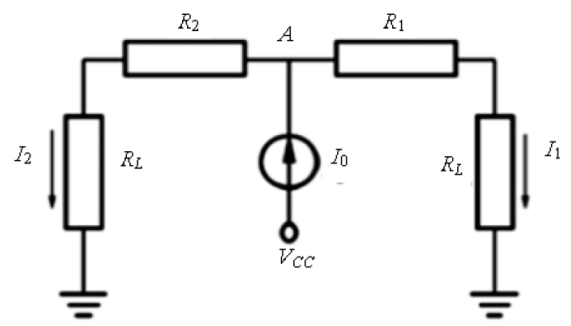

Fig. 2 Equivalent circuit.

Suppose that light is at point $A$ of the photosensitive layer on the optical displacement sensor, and the distance from the center is $X_{A}$. Let $\mathrm{P}$ layer resistance uniform. The equivalent resistances from point $A$ to the both sides of the optical displacement sensor are $R_{1}$ and $R_{2}$, the load resistance is $R_{L}$, and $R_{1}$ and $R_{2}$ are far greater than $R_{L}$. The distance between the poles is $2 L$, the currents flowing through the poles are $I_{1}$ and $I_{2}$, and the total photocurrent is $I_{0}$. According to the Lucovsky equation of the horizontal photoelectric effect, there is 


$$
I_{0}=I_{1}+I_{2}
$$

where

$$
\begin{aligned}
& I_{1}=\left[\left(L-X_{A}\right) I_{0}\right] /(2 L) \\
& I_{2}=\left[\left(L+X_{A}\right) I_{0}\right] /(2 L) .
\end{aligned}
$$

and

Therefore, it yields

$$
X_{A}=\left(I_{2}-I_{1}\right) L /\left(I_{2}+I_{1}\right) .
$$

If the currents $I_{1}$ and $I_{2}$ on the load resistance are measured, $X_{A}$ can be calculated.

When the photoelectric displacement sensor is adopted in the displacement measurement, the output voltage signal goes through the transmitter, and the output can reflect the size of the displacement. Through the $\mathrm{A} / \mathrm{D}$, digital signals of the displacement can be obtained. Because existing serious non-linearity between the output voltage and the measured displacement, it can be expressed as

$$
y=f(x)=a_{0}+a_{1} \frac{U}{U_{\max }}+a_{2}\left(\frac{U}{U_{\max }}\right)^{2}+a_{3}\left(\frac{U}{U_{\max }}\right)^{3}
$$

where $y$ is the measured weight; $U$ is the output voltage of the optical displacement sensor; $U_{\max }$ is the maximum output voltage of the optical displacement sensor; $a_{0}, a_{1}, a_{2}$, and $a_{3}$ are constant coefficients for the non-linear calibration. To eliminate the nonlinear error of the optical displacement sensor, the output $y$ of the optical displacement sensor could be sent to a compensator whose characteristic function is $z=F(y)$, where $F(y)=f^{-1}(y)$, and then the nonlinearity can be eliminated effectively. Obviously, $F(y)$ is a nonlinear function, and the output value $z$ after compensation is the same as measured $x$, thus the optical displacement sensor has ideal characteristics.

\subsection{Linearity}

One of the best characteristics of a transducer is considered to be linearity, that is, the output is linearly proportional to the input. The computation of linearity is done with reference to a straight line showing the relationship between the output and input. This straight line is drawn by using the method of least squares from the given calibration data. This straight line is sometimes called an idealized straight line expressing the input-output relationship. The linearity is simply a measure of the maximum deviation of any of the calibration points from this straight line.

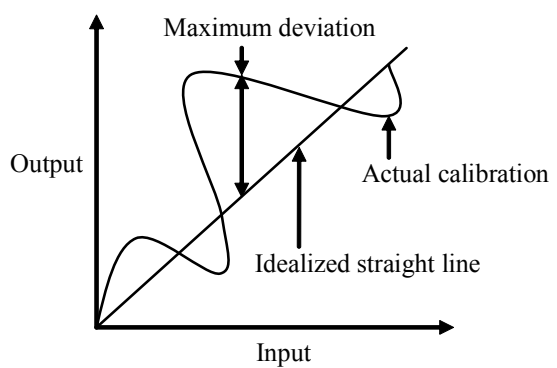

Fig. 3 Actual calibration curve.

Figure 3 shows the actual calibration curve i.e., a relationship between the input and output and a straight line drawn from the origin using the method of least squares.

\section{Percentageofnonlinearity $=$}

Maxdeviation from the idealized straight line Full scale deviation 100 .

Equation (4) expresses the nonlinearity as a percentage of full scale reading. It is desirable to keep the nonlinearity as small as possible as it would in that case result in small errors. In this research work, we have taken the performance of the optical displacement sensor. The experimental data obtained by conducting experiments on the sensor are given in Table 1. The output response curves of the sensor are shown in Fig. 4. It is clear that the output response of the pressure sensor shows the presence of nonlinearity.

Table 1 Experimental observations of the optical displacement sensor.

\begin{tabular}{c|c}
\hline Displacement $(\mathrm{mm})$ & Measured voltage $(\mathrm{V})$ \\
\hline 0.1 & 2.501 \\
\hline 0.2 & 2.511 \\
\hline 0.3 & 2.48 \\
\hline 0.4 & 2.482 \\
\hline 0.5 & 2.479 \\
\hline 0.6 & 2.479 \\
\hline 0.7 & 2.463 \\
\hline
\end{tabular}




\begin{tabular}{|c|c|}
\hline Displacement (mm) & Measured voltage (V) \\
\hline 0.8 & 2.464 \\
\hline 0.9 & 1.873 \\
\hline 1 & 1.567 \\
\hline 1.1 & 1.26 \\
\hline 1.2 & 1.278 \\
\hline 1.3 & 1.131 \\
\hline 1.4 & 0.987 \\
\hline 1.5 & 0.682 \\
\hline 1.6 & -0.322 \\
\hline 1.7 & -0.634 \\
\hline 1.8 & -0.922 \\
\hline 1.9 & -1.211 \\
\hline 2 & -1.125 \\
\hline 2.1 & -1.831 \\
\hline 2.2 & -2.184 \\
\hline 2.3 & -2.454 \\
\hline 2.4 & -2.455 \\
\hline 2.5 & -2.467 \\
\hline 2.6 & -2.474 \\
\hline 2.7 & -2.481 \\
\hline 2.8 & -2.499 \\
\hline 2.9 & -2.533 \\
\hline
\end{tabular}

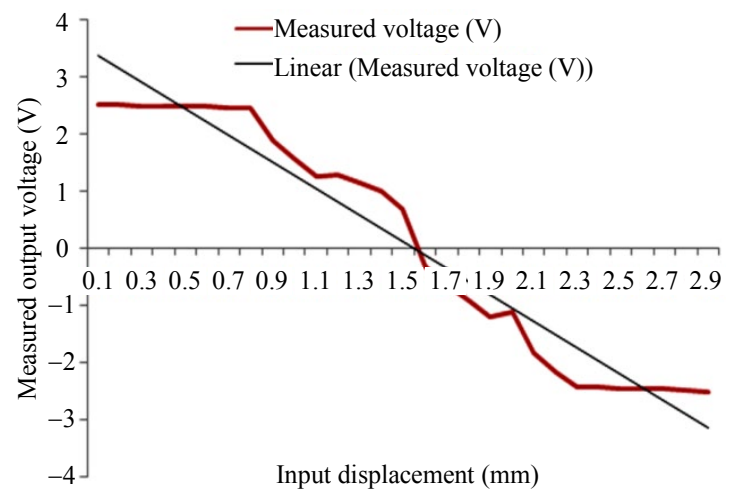

Fig. 4 Input-output characteristics of the optical displacement sensor.

The measured voltage and linear voltage are differentiated in the above figure. It has been observed from the above graph (Fig.4), in which the relation between the input displacement and voltage output of the optical displacement sensor is nonlinear. The following algorithm is used to compensate the nonlinearity of the sensor in this work.

\section{Extreme learning machine method}

The extreme learning machine method proposed by Huang et al. $[16,17]$ uses the single layer feed forward neural network (SLFN) architecture [18]. It randomly chooses the input weights and analytically determines the output weights of the SLFN. It has much better generalization performance with much faster learning speed. It requires less human interventions and can run thousands times faster than those conventional methods. It automatically determines all the network parameters analytically, which avoids trivial human intervention and makes it efficient in on-line and real-time applications. The extreme learning machine method has several advantages: the ease of use, faster learning speed, higher generalization performance, suitable for many nonlinear activation function and kernel functions.

\subsection{Single hidden layer feed-forward neural network}

The SLFN function with $L$ hidden nodes [19], [20] can be represented as a mathematical description of the SLFN incorporating both additive and radial basis function (RBF) hidden nodes in a unified way is given as follows:

$$
f_{L}(x)=\sum_{i=1}^{L} \beta_{i} G\left(a_{i}, b_{i}, x\right), x \in R^{n}, a_{i} \in R^{n}
$$

where $a_{i}$ and $b_{i}$ are the learning parameters of hidden nodes, and $\beta_{i}$ is the weight connecting the $i$ th hidden node to the output node. $G\left(a_{i}, b_{i}, x\right)$ is the output of the $i$ th hidden node with respect to the input $x$. For the additive hidden node with the activation function $g(x): R \rightarrow R$ (e.g. sigmoid and threshold), $G\left(a_{i}, b_{i}, x\right)$ is given by

$$
G\left(a_{i}, b_{i}, x\right)=g\left(a_{i}, x+b_{i}\right), b_{i} \in R
$$

where $a_{i}$ is the weight vector connecting the input layer to the $i$ th hidden node, and $b_{i}$ is the bias of the $i$ th hidden node. $\left(a_{i}, x\right)$ denotes the inner product of vector $a_{i}$ and $x$ in $R^{n}$.

For $N$ arbitrary distinct samples, $\left(x_{i}, t_{i}\right) \in R^{n} \times$ 
$R^{m}$. Here, $x_{i}$ is an $n \times 1$ input vector, and $t_{i}$ is an $m \times 1$ target vector. If an SLFN with $L$ hidden nodes can approximate these $N$ samples with zero error, then it implies that there exists $\beta_{i}, a_{i}$, and $b_{i}$ such that

$$
f_{L}\left(x_{j}\right)=\sum_{i-1}^{L} \beta_{i} G\left(a_{i}, b_{i}, x_{j}\right) . \quad j=1,2, \cdots, N
$$

The above equation can be written as

$$
H \beta=T
$$

where

$$
\begin{gathered}
H(\bar{a}, \bar{b}, \bar{x})=\left(\begin{array}{l}
G\left(a_{1}, b_{1}, x_{1}\right) \cdots G\left(a_{L}, b_{L}, x_{1}\right) \\
G\left(a_{1}, b_{1}, x_{N}\right) \cdots G\left(a_{L}, b_{L}, x_{N}\right)
\end{array}\right) \\
N \times L
\end{gathered}
$$

with $\tilde{a}=a_{1}, \cdots, a_{L} ; \tilde{b}=b_{1}, \cdots, b_{L} ; \tilde{x}=x_{1}, \cdots, x_{N}$,

$$
\beta=\left[\begin{array}{c}
\beta_{1}^{T} \\
\vdots \\
\beta_{L}^{T}
\end{array}\right] L \times m \text { and } T=\left[\begin{array}{c}
T_{1}^{T} \\
\vdots \\
T_{L}^{T}
\end{array}\right] N \times m .
$$

$\mathrm{H}$ is the hidden layer output matrix of the SLFN with $i$ th column of $\mathrm{H}$ being the $i$ th hidden node's output with respect to inputs $x_{1}, x_{2}, \cdots, x_{N}$.

From the observed readings of the optical displacement sensor shown in Table 1, the simulation study has been carried out, and the following results have been obtained.

The results obtained by ELM based nonlinearity compensation of the optical displacement sensor are listed in Table2. Three different activation functions namely sine, sigmoid, and radial bases are used here. The training time, testing time, and root mean square error (RMSE) values are tabulated. Different numbers of hidden nodes are assigned for the ELM algorithm. 100 trials have been conducted for the algorithm, and the average results are shown in Table 2. It can be seen from Table 2 that the ELM learning algorithm spends 0 -second CPU time obtaining the testing RMSE of 0.0033 with the sine activation function, 0.0156 -second $\mathrm{CPU}$ time obtaining the RMSE value of 0.0030 with the sigmoid activation function, and 0.0780 -second CPU time obtaining the RMSE value of 0.0011 . The

\begin{tabular}{|c|c|c|c|c|c|}
\hline $\begin{array}{l}\text { Activation } \\
\text { function }\end{array}$ & $\begin{array}{c}\text { No. of } \\
\text { generations }\end{array}$ & $\begin{array}{c}\text { No. of } \\
\text { hidden } \\
\text { nodes }\end{array}$ & $\begin{array}{l}\text { Training time } \\
\quad(\mathrm{sec})\end{array}$ & $\begin{array}{l}\text { Testing } \\
\text { time (s) }\end{array}$ & RMSE \\
\hline \multirow{11}{*}{ sine } & \multirow{11}{*}{100} & 1 & 0.0156 & 0 & 0.0415 \\
\hline & & 2 & 0 & 0 & 0.0333 \\
\hline & & 3 & 0 & 0 & 0.0219 \\
\hline & & 4 & 0 & 0 & 0.0057 \\
\hline & & 5 & 0 & 0 & 0.0053 \\
\hline & & 10 & 0.0156 & 0 & 0.0044 \\
\hline & & 15 & 0 & 0 & 0.0042 \\
\hline & & 20 & 0.0312 & 0.0156 & 0.0033 \\
\hline & & 25 & 0 & 0 & 0.0033 \\
\hline & & 30 & 0 & 0 & 0.0033 \\
\hline & & 50 & 0 & 0 & 0.0033 \\
\hline \multirow{9}{*}{ sigmoid } & \multirow{9}{*}{100} & 1 & 0 & 0 & 0.6315 \\
\hline & & 2 & 0.0156 & 0.0156 & 0.0298 \\
\hline & & 3 & 0 & 0 & 0.0292 \\
\hline & & 4 & 0.0156 & 0.0156 & 0.0056 \\
\hline & & 5 & 0 & 0 & 0.0053 \\
\hline & & 10 & 0 & 0 & 0.0044 \\
\hline & & 15 & 0 & 0 & 0.0032 \\
\hline & & 20 & 0.0156 & 0.0156 & 0.0031 \\
\hline & & 25 & 0.0156 & 0 & 0.0030 \\
\hline \multirow{7}{*}{ radial basis } & \multirow{7}{*}{100} & 2 & 0.0780 & 0.0156 & 0.0420 \\
\hline & & 3 & 0.0624 & 0 & 0.0051 \\
\hline & & 4 & 0.0780 & 0 & 0.0050 \\
\hline & & 5 & 0.0624 & 0 & 0.0050 \\
\hline & & 10 & 0.0780 & 0 & 0.0044 \\
\hline & & 15 & 0.0936 & 0 & 0.0012 \\
\hline & & 20 & 0.0780 & 0 & 0.0011 \\
\hline
\end{tabular}
new ELM runs 170 times faster than the conventional BP algorithms.
Table 2 Results of ELM based nonlinearity compensation of the optical displacement sensor.

\section{Results}

A computer simulation was carried out in the MATLAB 12 environment using an experimental dataset. The experimental data were collected from the optical displacement sensor shown in Table 1. The observed simulation results with different activation functions and hidden nodes before and after compensation of nonlinearity are shown in Figs. $5-10$. Initially, the numbers of hidden nodes were assumed to be 1 and 5 with the sine activation function as shown in Figs. 5 and 6. It showed better linearity characteristics. Then, the hidden nodes 
were increased with different activation functions namely the sigmoid function as shown in Figs. 7 and 8, the radial basis function as shown in Fig. 9, and the triangular basis function as shown in Fig. 10. It was observed that the ELM model yielded the lowest training time of zero second to obtain better linearity in the overall response. At the same time, it yielded the lowest RMSE value of 0.0011 with the radial basis activation function. From the output response graphs, the presence of nonlinearity in the sensor was compensated by the use of this algorithm.

ELM based nonlinearity compensation

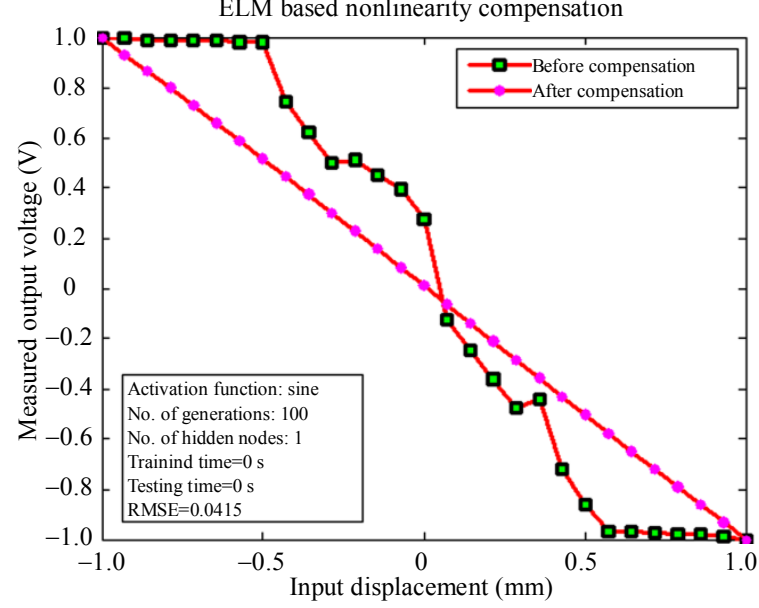

Fig. 5 ELM based nonlinearity compensation with the sine activation function.

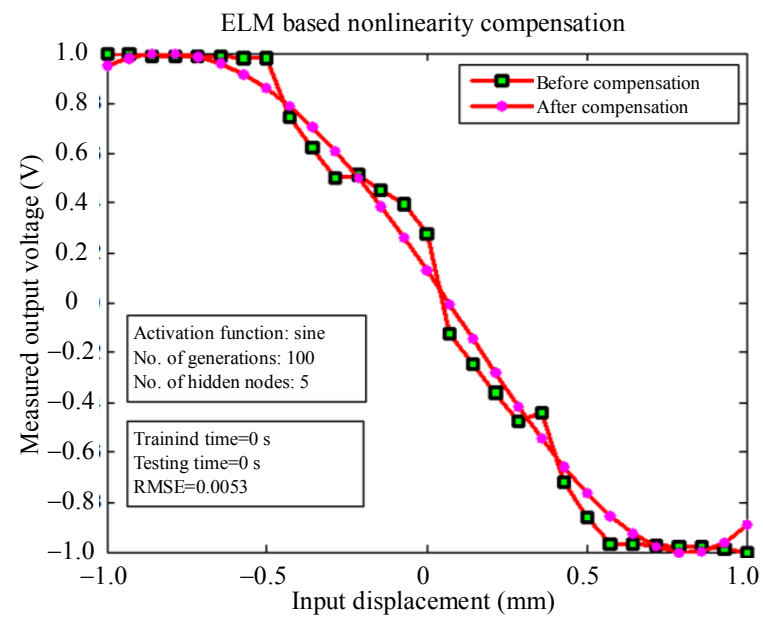

Fig. 6 ELM based nonlinearity compensation with the sine activation function.

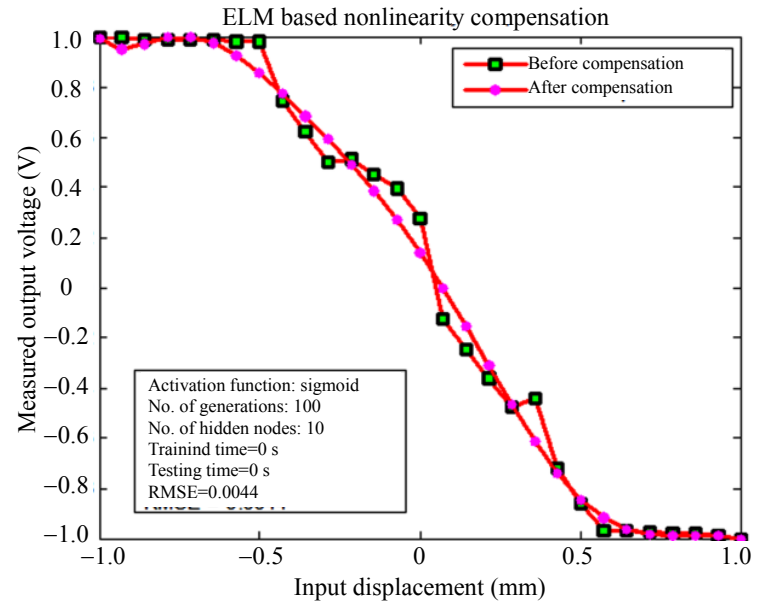

Fig. 7 ELM based nonlinearity compensation with the sigmoid activation function.

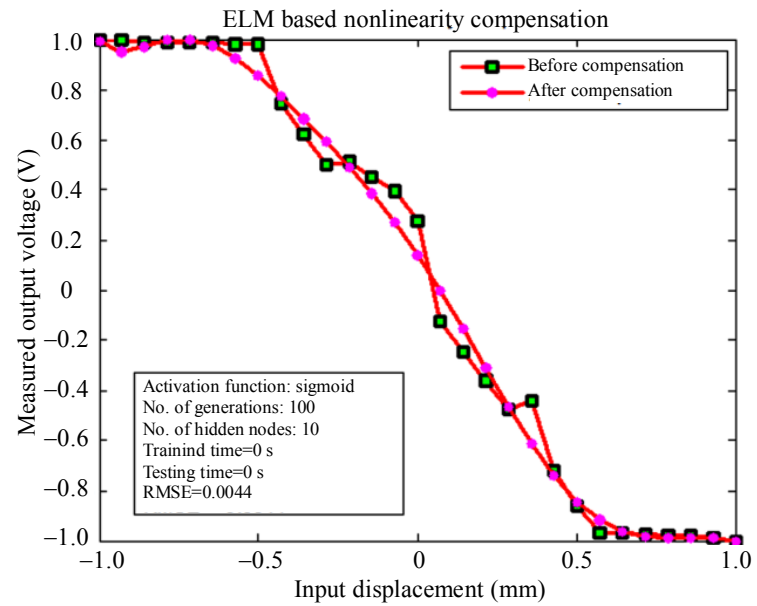

Fig. 8 ELM based nonlinearity compensation with the sigmoid activation function.

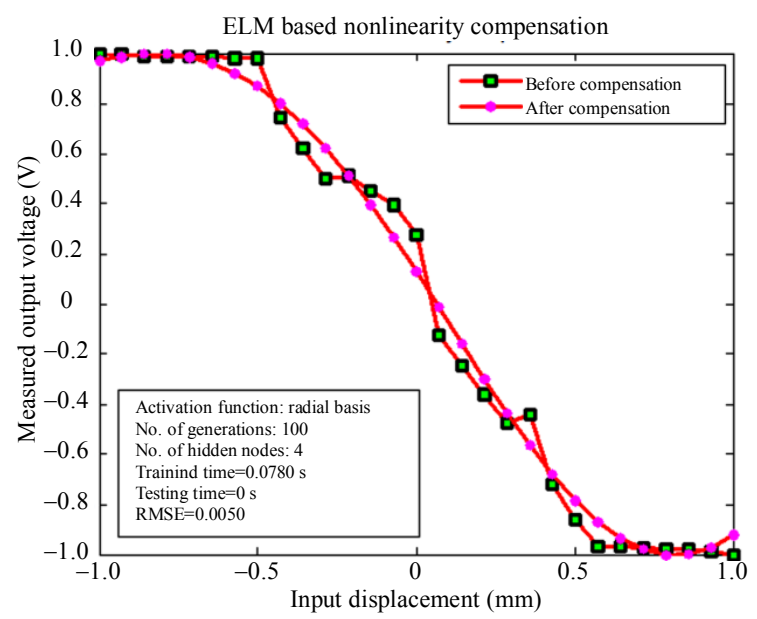

Fig. 9 ELM based nonlinearity compensation with the radial basis activation function. 


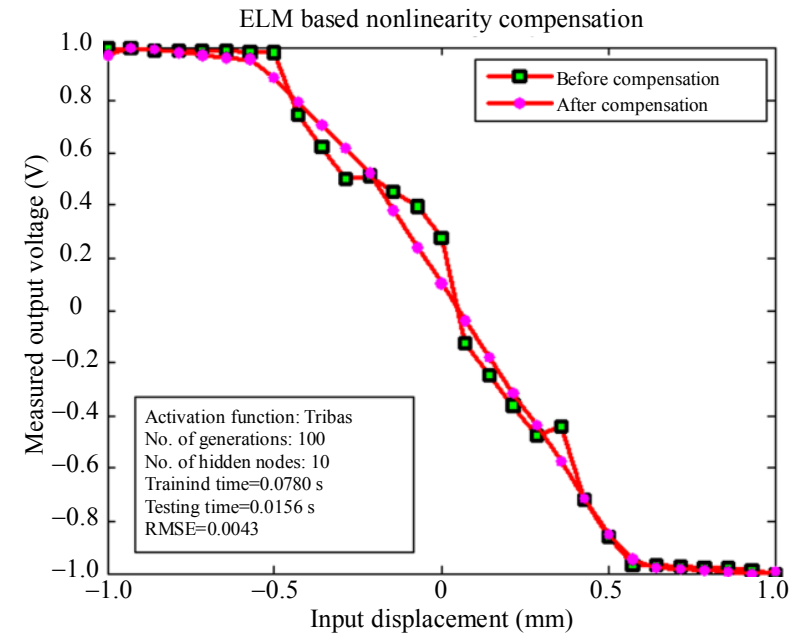

Fig. 10 ELM based nonlinearity compensation with the triangular basis activation function.

\section{Conclusions}

An ELM method was proposed to adaptively compensate for the nonlinearity offered by the optical displacement sensor. The ELM method based nonlinearity compensation produces the less training time and better RMSE value when comparing to others. Results revealed that the ELM method had given the best linearization approximation and compensated the nonlinearity with very less training time and the lowest MSE among the proposed tools. The proposed algorithm offers a less complexity structure and is simple in the testing and validation procedure. This adaptive algorithm can also be applied to any sensor having a nonlinear characteristic. This hybrid technique is used to make a sensor output as more linear as possible. Further, this adaptive algorithm is preferable for real-time implementation also.

\section{Acknowledgement}

The authors would like thank the management of Einstein College of Engineering, Tirunelveli and Ultra College of Engineering and Technology for women, Madurai for providing us the support to publish this completion of research work.

Open Access This article is distributed under the terms of the Creative Commons Attribution License which permits any use, distribution, and reproduction in any medium, provided the original author(s) and source are credited.

\section{References}

[1] J. Xu, D. Peng, W. Wan, and W. Yang, "New way of producing electrical traveling wave signal based on photo electricity technology in design of time-grating displacement sensor," Chinese Journal of Sensors and Actuators, 2007, 20(3): 532-535.

[2] T. Xu, H. Lu, and W. Luo, "A robust photoelectric angular position sensor especially for a steerable underground boring tool original," Sensors and Actuators A: Physical, 2005, 120(2): 311-316.

[3] Y. Yu, M. Ding, and L. Zhang, "The algorithmic analysis of linear approximation for characteristic region of displacement sensor," Chinese Journal of Sensors and Actuators, 2010, 23(6): 840-843.

[4] Z. Shi, J. Kang, and R. Sun, "BP NN-based method for lens distortion correction of large-field imaging," Optics and Precision Engineering, 2005, 13(3): 348-353.

[5] W. Ling, Z. Wang, and F. Gao, "Real-time digital correction for distortion in photo electronic measuring system," Optics and Precision Engineering, 2007, 15(2): 277-282.

[6] Y. Qiao, F. Gao, Z. Wang, Y. Zhao, and J. Li, "Distortion correction for the photoelectricity measuring system based on the cubic fitting equation," Opto-Electronic Engineering, 2008, 35(6): 28-31.

[7] D. Yu, Z. Su, and K. Yan, "A new type of machine vision systems with algorithm for image correction," Laser \& Infrared, 2008, 38(11): 1173-1176.

[8] X. Bai, S. Cai, F. Gao, Y. Qiao, and M. Dai, "Distortion correction for photo electric measuring system based on BP neural network," Laser \& Infrared, 2010, 40(1): 79-82.

[9] S. Cai, Q. Li, and Y. Qiao, "Camera calibration of attitude measurement system based on BP neural network," Journal of Optoelectronics Laser, 2007, 18(7): 832-834.

[10] Z. Wang, Y. Li, L. Lou, H. Wei, and Z. Wang, "Application of BP neural network to nonlinearity correction of optical tweezers," Optics and Precision Engineering, 2008, 16(1): 6-10.

[11] F. X. Zheng and S. P. Ji, "An improved non uniformity correction algorithm for IRFPA based on neural network," Laser \& Infrared, 2008, 38(9): 937-938.

[12] V. Vapnik, Statistical learning theory. New York: John Wiley \& Sons Inc., 1998: 12-35.

[13] J. Q. E, Intelligent fault diagnosis and its application. 
Changsha: Hunan University Press, 2006: 70-130.

[14] A. T. C. Goh and S. H. Goh, "Support vector machines: their use in geotechnical engineering as illustrated using seismic liquefaction data," Computers and Geotechnics, 2007, 34(5): 410-421.

[15] X. Jiang, Z. Yi, and J. Lv, "Fuzzy SVM with a new fuzzy membership function," Neural Computing and Application, 2006, 15(2): 268-276.

[16] G. Huang, L. Chen, and C. Siew, "Universal approximation using incremental constructive feed forward networks with random hidden nodes," IEEE Transactions on Neural Networks, 2006, 17(4): 879-892.

[17] N. Liang, G. Huang, P. Saratchandran, and N. Sundararajan, "A fast and accurate on-line sequential learning algorithm for feed forward networks," IEEE Transactions on Neural Networks, 2006, 17(6): 1411-1423.

[18] A. J. Annema, K. Hoen, and H. Wallinga, "Precision requirements for single-layer feed forward neural networks", In Fourth International Conference on Microelectronics for Neural Networks and Fuzzy Systems, The Netherlands, pp: 145-151, 1994.

[19] G. Huang, Q. Zhu, and C. Siew, "Extreme learning machine: a new learning scheme of feed forward neural networks," in International Joint Conference on Neural Networks, Singapore, pp: 985-990, 2004.

[20] G. B. Huang, Q. Y. Zhu, and C. K. Siew, "Extreme learning machine: theory and applications," Neurocomputing, 2006, 70(1-3): 489-501. 